\title{
High-velocity microsprays enhance antimicrobial activity in $S$. mutans biofilms
}

S. Fabbri ${ }^{1}$, D.A. Johnston ${ }^{2}$, A. Rmaile ${ }^{3}$, B. Gottenbos ${ }^{3}$, M. De Jager ${ }^{3}$, M. Aspiras ${ }^{4}$, E. M. Starke $^{5}$, M. T. Ward ${ }^{5}$ and P. Stoodley ${ }^{1,6}$

1 National Centre for Advanced Tribology at Southampton (nCATS), University of Southampton, Southampton SO17 1BJ, United Kingdom.

${ }^{2}$ Biomedical Imaging Unit, School of Medicine, University of Southampton, Southampton SO16 6YD, UK.

${ }^{3}$ Philips Research, High Tech Campus, Eindhoven 5656 AE, The Netherlands.

${ }^{4}$ Wrigley, Chicago, Illinois 60613, USA.

${ }^{5}$ Philips Oral Healthcare, Bothell, Washington 98021, USA.

${ }^{6}$ Department of Microbial Infection and Immunity and the Department of Orthopaedics, Centre for Microbial Interface Biology, The Ohio State University, Columbus, Ohio 43210, USA.

Corresponding author:

Ms Stefania Fabbri

National Centre for Advanced Tribology at Southampton (nCATS)

Engineering and the Environment, University of Southampton

Highfield Campus, Southampton SO17 1BJ

Email: stefaniafabbri1987@gmail.com

Phone: +447895314989 
KEYWORDS: Plaque/Plaque biofilms, Dental hygiene, Antimicrobials/Antimicrobial resistance, Caries detection/diagnosis/prevention, Oral hygiene, Drug delivery

\section{ABSTRACT}

Streptococcus mutans in dental plaque biofilms play a role in caries development. The biofilm's complex structure enhances the resistance to antimicrobial agents by limiting the transport of active agents inside the biofilm. We assessed the ability of high-velocity water microsprays to enhance delivery of antimicrobials into 3-days old $S$. mutans biofilms. Biofilms were exposed to a $90^{\circ}$ or $30^{\circ}$ impact, firstly using a $1-\mu \mathrm{m}$ tracer beads solution $\left(10^{9}\right.$ beads $/ \mathrm{mL}$ ) and secondly, a $0.2 \%$ Chlorhexidine (CHX) or $0.085 \%$ Cetylpyridinium chloride (CPC) solution. For comparison, a 30-sec diffusive transport and simulated mouthwash were also performed. Confocal microscopy was used to determine number and relative bead penetration depth (RD) into the biofilm. Assessment of antimicrobial penetration was determined by calculating the killing depth $(\mathrm{KD})$ detected by live/dead viability staining. We firstly demonstrated that the microspray was able to deliver significantly more microbeads deeper in the biofilm compared to diffusion and mouthwashing exposures. Next our experiments revealed that the microspray yielded better antimicrobial penetration evidenced by deeper killing inside the biofilm and a wider killing zone around the zone of clearance than a diffusion transport with the same antimicrobials. Interestingly the $30^{\circ}$ impact in the distal position delivered approximately 16 times more microbeads and yie lded approximately $20 \%$ more bacteria killing (for both $\mathrm{CHX}$ and CPC) than the $90^{\circ}$ impact. These data suggest that high-velocity water microsprays can be used as an effective mechanis $m$ to deliver microparticles and antimicrobials inside $S$. mutans biofilms. High shear stresses generated at the biofilm/burst interface might have enhanced beads and antimicrobials delivery inside the remaining biofilm by combining forced advection into the biofilm matrix and physical restructuring of the biofilm itself. Further, the impact angle has potential to be optimized both for biofilm removal and active agents' delivery inside biofilm in those protected areas where some biofilm might remain. 


\section{INTRODUCTION}

Oral biofilms play an important role in the development and the persistence of caries, halitosis, gingivitis and periodontitis (Marsh 2004). The addition of antiplaque agents or antimicrobials to toothpastes, mouthwashes and varnishes in order to kill bacteria is one of the most common ways to control oral diseases (Marsh 2006). The challenge, however, is that dental plaque bacteria organize themselves into biofilms, which increases their tolerance to these active agents through diffusion limitation (Stoodley et al. 2008; von Ohle et al. 2010). The role of the hydrodynamics in the enhancement of the delivery of active agents inside the biofilm has become a topic of interest, since it might also be utilized to improve delivery of dentifrices to oral surfaces (teeth, gums, tongue), or to bacteria directly. Fluiddynamic activity generated by powered toothbrushes can enhance antimicrobials/antiplaque agents delivery into remaining biofilm compared to simple diffusional transport (He et al. 2014; Jongsma et al. 2015; Stoodley et al. 2007). Microsprays are a useful strategy for removal since they require low liquid volume but also have an air/water interface moving over the solid surface which facilitates biofilm removal. We previously investigated the ability of high-velocity water microsprays to mechanically remove interproximal biofilms (Rmaile et al. 2014; Rmaile et al. 2015) and discovered that the biofilm was physically "churned up" during the exposure (Fabbri et al. 2016). We hypothesized that this churning of the biofilm might improve the delivery of active agents into the biofilm extracellular polymeric substance (EPS) matrix. To test this hypothesis, we assessed the potential of highvelocity water microsprays for delivery into in vitro Streptococcus mutans biofilms, firstly,

us ing 1- $\mu \mathrm{m}$-diameter fluorescent beads as tracer particles, and, secondly, using Chlorhexidine (CHX) and Cetylpyridinium chloride (CPC), two antimicrobials commonly found as active ingredients in dentifrices. Delivery of these materials into the biofilm by microspray was compared with a static (diffusion only) delivery and simulated swirling mouthwash.

\section{MATERIALS AND METHODS}

\section{$\underline{\text { Biofilm growth conditions }}$}

Biofilms were grown on glass microscope slides (75 mm x $25 \mathrm{~mm}$, Corning, Sigma-Aldrich). The slides were first conditioned with $10 \mathrm{~mL}$ of $2 \%$ sucrose-supplemented brain-heart infusion $(\mathrm{BHI}+\mathrm{S})$ and $1 \%$ type II porcine gastric mucin (Sigma-Aldrich) (BHI+SM) to 
simulate salivary proteins and establish a conditioning film. The slides were placed in a petri plate and inoculated with a $S$. mutans UA159 adjusted overnight culture $\left(10^{6} \mathrm{cfu} / \mathrm{mL}\right)$ and grown in $10 \mathrm{~mL} \mathrm{BHI+SM} \mathrm{medium,} \mathrm{then,} \mathrm{grown} \mathrm{under} \mathrm{static} \mathrm{conditions} \mathrm{for} 72 \mathrm{~h}$ at $37{ }^{\circ} \mathrm{C}$ and $5 \% \mathrm{CO}^{2}$ with medium replacement every $24 \mathrm{~h}$.

\section{Microbeads delivery}

Carboxylate-modified polystyrene yellow/green-fluorescent beads $\left(\lambda_{\text {ex }} / \lambda_{\text {em }}: 470 / 505\right.$, density= $2.5 \%$, charge density $\geq 0.008 \mathrm{mEq}$, Sigma-Aldrich (L4655)), of 1 - $\mu \mathrm{m}$-diameter, stock solution was diluted down to $10^{9}$ beads $/ \mathrm{mL}$. A Philips Sonicare AirFloss (AF), a commercially available oral hygiene device for interdental cleaning, was used to generate high-velocity water microsprays. The device delivers a volume of $130 \pm 0.03 \mu \mathrm{L}(\mathrm{n}=11)$ as a microspray in a single discrete shot. The device was filled with $3 \mathrm{~mL}$ of the beads working solution; therefore approximately $1.3 \times 10^{8}$ beads were delivered in each shot. First, $S$. mutans biofilm-covered slides were exposed to a single microspray shot at either a $90^{\circ}$ or a $30^{\circ}$ impact angle with the tip held a distance of $5 \mathrm{~mm}$ from the biofilm. For comparative purposes the delivery of beads into the biofilm was compared by 1) diffusion alone in a static incubation by gently adding $130 \mu \mathrm{L}$ of bead solution $\left(1.3 \times 10^{8}\right.$ beads $)$ over the biofilm and let to diffuse for 30 seconds; or 2) by a simulated swirling mouthwash exposure to beads in an overlying liquid by placing the glass slide inside a petri plate filled with $3 \mathrm{~mL}$ of bead solution and then shaken for 30 secs at $200 \mathrm{rpm}$. Three independent replicates were performed for each experiment. Immediately after each experiment (microspray, static and shaking), the slide samples were washed once with 1\% Phosphate Buffered Saline (PBS) solution to removing loose beads. One PBS tablet (Sigma-Aldrich) was dissolved in $200 \mathrm{~mL}$ distilled water to obtain $10 \mathrm{mM}$ phosphate buffer, $2.7 \mathrm{mM}$ potassium chloride and $137 \mathrm{mM}$ sodium chloride.

\section{Quantification of beads in the biofilm}

The penetration of beads into the biofilm was quantified by confocal microscopy and image analysis. After exposure, the biofilms were fixed with 4\% Paraformaldehyde (PFA) solution to preserve structure (von Ohle et al. 2010). The fixed biofilms were stained with the nucleic acid stain Syto 63 (Invitrogen) to visualize the total biomass and subsequently imaged under Confocal Laser Scanner Microscope (CLSM, Leica TCS SP5). A non-treated biofilm was used as control. Confocal 3D stacks were collected for each of three independent replicates 
for the non-treated, static, shaking and microspray experiments. For the biofilms exposed to the microsprays we took confocal images from three locations 1 to $2 \mathrm{~mm}$ outside of the clearance zone caused by the microspray (Supplemental material 1, Figure 1A, Appendix Figure 1A-B). For the $30^{\circ}$ shot we distinguished between the proximal area and distal areas with respect to distance from the device nozzle. For the non-treated, static and shaking exposures we collected data at 3 random positions on the slide (Supplemental material 1, Appendix Figure 1C). To quantify the penetration of the beads into the biofilm we used a relative depth ratio ( $R D_{\text {BEADS }}$ ) to account for differences in biofilm thickness at each $\mathrm{XY}$ pixel location on the substratum, as explained previously (Miller et al. 2013) (Supplemental material 2).

\section{Antimicrobial delivery and killing depth in the biofilm}

To assess the ability of the micros pray to enhance the delivery and killing of bacteria within the biofilm by antimicrobial agents we used CHX and CPC (Sigma-Aldrich). The stock solutions were diluted in $1 \% \mathrm{PBS}$ to $0.2 \%$ (wt/vol) $\mathrm{CHX}$ and $0.085 \%$ (wt/vol) $\mathrm{CPC}$. The AF device was filled with $3 \mathrm{~mL}$ of the CHX or CPC solutions. PBS (1\%) alone was used as a control. The biofilms were then exposed to a single $90^{\circ}$ or a $30^{\circ}$ impact angle microspray shot or a static incubation for each antimicrobial and the control. Simulated mouthwash (shaking experiments) were also performed using CHX. Static diffusion incubation was also performed with 1\% PBS alone as a non-treated control. Three independent replicates were performed for each experiment. The influence of the various exposures on biofilm structure, depth of killing and the zone of lateral killing (in the case of the microspray exposures) were assessed by confocal microscopy. Immediately after exposure the biofilms were stained with Live/Dead stain (BacLight, Invitrogen) according to manufacturer's instructions. The stained samples were then rinsed with PBS to remove excess stain and immediately imaged under CLSM. Live cells were stained green and dead and membrane compromised cells were stained red (von Ohle et al. 2010). Confocal 3D stacks were taken on each of three independent replicate for the static, the shaking and microspray experiments in the same way as they were taken for the beads experiments (Supplemental material 1, Appendix Figure 1A-C). Since we could not directly visualize the antimicrobial age nt within the biofilm, we used the depth of bacterial killing (measured from live/dead staining) as a relative indicator of antimicrobial penetration depth as described elsewhere (He et al. 2014) (Supplemental 
material 3). This will underestimate the absolute penetration depth since it more closely relates to the depth where the microbial biocidal concentration (MBC) was achieved, however for practical purposes the depth of killing is arguably more relevant. In addition to measuring the depth of killing for those biofilms exposed to the microsprays, we also assessed the proportion of bacteria killed as a function of lateral distance from the edge of the zone of clearance by measuring the variation in the red/green signal ratio (Supplemental material 4). The lateral extent of the antimicrobial killing zone from the edge of the impact clearance zone for each antimicrobial was defined as the distance for which $R / G$ was greater than 1.5 (He et al. 2014).

\section{$\underline{\text { Statistical analysis }}$}

Statistical comparisons were performed using analysis of variance (ANOVA) with a Bonferroni post hoc test. Differences were considered significant for $\mathrm{P}<0.05$.

\section{RESULTS}

\section{$\underline{\text { Biofilm structure, thickness, and bead penetration }}$}

The untreated (control) biofilm was relatively uniform with a slightly undulating surface and an average thickness of $51.8 \pm 4.9 \mu \mathrm{m}$ (Table 1, Figure 1B). The static incubation with the beads did not significantly change biofilm structure and thickness (Table 1, Figure 1C). However, the shaking exposure to the beads resulted in some biofilm loss, probably due to shear stresses.

CLSM images of the biofilms exposed to microbeads showed more beads were present in the biofilm after exposure to the microspray (both the $30^{\circ}$ and the $90^{\circ}$ impact angles) than after their introduction by static or shaking means (Figure 1C-D). Beads were observed in the confocal cross-sections images, confirming their penetration inside the biofilm (Figure 1EF). Figure 2 shows the beads distribution inside the biofilm in each relative depth "slice" (0$25 \%, 25-50 \%, 50-75 \%$ and $75-100 \%)$. In all cases the $30^{\circ}$ impact angle delivered significantly more beads than the $90^{\circ}$ impact angle microspray and both $\left(90^{\circ}\right.$ and $\left.30^{\circ}\right)$ yielded a significantly higher beads penetration than the shaking or static incubations. In addition, the $30^{\circ}$ impact angle microspray delivered significantly more beads in the in the distal zone than in the proximal zone. 
$\underline{\text { Biofilm structure, thickness, and antimicrobial killing depth }}$

Static treatment with CHX, CPC and PBS had no significant effect on biofilm thickness compared to the non-treated biofilm (Table 1) but shaking exposure to $\mathrm{CHX}$ significantly $(\mathrm{P}<0.05)$ reduced biofilm thic kness to $43.6 \pm 1.9 \mu \mathrm{m}$ compared to the biofilm exposed to static diffusion using the same active agent (Table 1). The biofilm thickness after the microspray exposure (both $30^{\circ}$ and $90^{\circ}$ impact angles) to $\mathrm{CHX}, \mathrm{CPC}$ and $\mathrm{PBS}$ was significantly less than the thickness after the static exposure to the same antimicrobials.

The $30^{\circ}$ and $90^{\circ}$ impact angles microspray caused a greater depth of killing with both antimicrobials than achieved with the simulated mouthwash shaking and static incubation (Figure 3A-B). In addition, when the biofilm was exposed to $30^{\circ}$ impact angle microspray, both antimicrobials generated a significantly greater killing depth in the distal position than in the proximal position . Biofilm exposed to a static assay showed that CPC resulted in greater killing depth than CHX (Figure3C). No dead zones were observed in the biofilm samples after the static diffusion assay performed with PBS (Figure 3C). Surprisingly, the microspray (both $90^{\circ}$ and $30^{\circ}$ impact angles) performed with PBS alone resulted in a zone of killing in the upper part of the biofilm, however, this was significantly lower than that achieved with the antimicrobials (Figure 3C).

Cross-sections of confocal images of biofilms samples exposed to microsprays and static assay using $\mathrm{CHX}, \mathrm{CPC}$ and PBS were in agreement with our results (Figure 4). In particular, biofilms samples exposed to $\mathrm{CHX}$ and $\mathrm{CPC}$ revealed that both $30^{\circ}$ and $90^{\circ}$ impact angle microsprays caused an increase in the size of the red areas (dead biofilm) compared to static transport (Figure4A-H). In addition, no dead zones were observed in the PBS-treated samples as shown by an increase of the green areas (live biofilm, Figure 4I-L).

Finally, the $30^{\circ}$ and $90^{\circ}$ impact angle microsprays caused a higher lateral zone of killing (up to $200 \mu \mathrm{m}$ from the ZOC edge) with both antimicrobials than the same experiments performed with PBS ( $52 \mu \mathrm{m}$ ) (Appendix Figure 2A-B). No dead zones were observed in the PBS controls (Appendix Figure 2C).

\section{DISCUSSION}

The purpose of this study was to assess whether a high-velocity water microspray enhances the penetration of antimicrobials into biofilms using a laboratory-grown $S$. mutans biofilm. 
While human dental plaque is clearly more complex in terms of microbial diversity (Dewhirst et al. 2010), than the single-specie $S$. mutans biofilm, the sticky glucans produced by glucosyltransferases released by $S$. mutans has a significant determination of the mechanical stability, ecological succession, development of acidic and anaerobic gradients of cariogenic biofilm, even if present in relatively low numbers (Bowen and Koo 2011; Koo et al. 2013). As such, $S$. mutans biofilms have been widely used in dental and microbiology research as a biofilm models for the study of caries, biofilm mechanical properties and detachment (Hashizume and Dariva 2015; Hwang et al. 2014; Vinogradov et al. 2004).

Firstly, we demonstrated that the microspray was able to deliver significantly more microbeads deeper in the biofilm compared to a simple diffusion and a simulated mouthwash (Figure 2). Next, we confirmed that the microspray yielded better penetration of CHX and CPC evidenced by a deeper bacteriostatic effect within the biofilm (Figure 4, Error! Reference source not found.) and a wider killing zone around the zone of clearance (Appendix Figure 2). The microspray used in our experiments was a turbulent complex twophase burst of water and air which lasted $60 \mathrm{~ms}$ (Fabbri et al. 2016). Once the spray impacts the surface, the flow can be divided in two regions: an impingement region in the centre of the impact followed by a two-phase wall-jet region where the flow spreads radially outward. Shear stresses generated at the jet/film interface rise as a function of radial distance, reaching a maximum value before decreasing again afterwards (Deshpande and Vaishnav 1983; Phares et al. 2000). Our data suggests that the stress generated is not only responsible for the biofilm detachment but also for structural deformations (what we define as biofilm "churning up") of the unre moved biofilm at edges of the clearance zone. In addition, studies on rinsing flows, where a water jet impinges on a flat surface coated with a second fluid at higher viscosity, demonstrated the formation of recirculation zones downstream at the interface between the two liquids (Hsu et al. 2011; Walker et al. 2012). The formation of eddies at the edges of the clearance zone could have contributed to the increased mixing between biofilm and the beads or antimicrobial solutions. Interestingly, a $30^{\circ}$ nozzle inclination was more powerful (in terms of beads delivery and bacterial killing caused by the antimicrobials) in the distal part of the exposed area compared to the proximal part and to a $90^{\circ}$ microspray. In the $30^{\circ}$ microspray, there would be an expected increase in the velocity in the direction of shooting (i.e., distal position) and a reduction in the opposite direction (i.e., proximal position). Such an asymmetrical velocity distribution may "focus" the impact and create cohesive failure at 
the biofilm-substratum interface (i.e. the low impact angle allows the spray to go between the biofilm-substratum interface in the distal position which could enhance beads and dentifrice penetration.

After the static experiment only 925 beads per $\mathrm{cm}^{2}$ penetrated $30 \%$ of the biofilm depth. Previous studies in biofilm permeability using fluorescent beads are consistent with our data showing that the beads penetration was limited to the outer biofilm layers $(20-30 \%$ of the biofilm thickness) (Drury et al. 1993a; Drury et al. 1993b) or needed timescales up to hours to reach up 90-95\% biofilm substratum by diffusion alone (Miller et al. 2013), in which case some of the effect could be "overgrowth" of the biofilm as seen by Chew et al. (Chew et al. 2014). The same trend was observed for the antimicrobial experiments where the bacterial killing depths yielded by CPC and $\mathrm{CHX}$ after a simple diffusion transport were approximately $5 \%$ and $20 \%$ respectively. These data are consistent with previous studies on oral biofilms showing that CHX and CPC antimicrobial efficacy decreased with increasing biofilm depth (Hope and Wils on 2004; von Ohle et al. 2010) with penetration times up to 20 mins to reach half of the biofilm thickness (Corbin et al. 2011; Nance et al. 2013). It is well known that the biofilm matrix confers antimicrobial tolerance (Costerton et al. 1999). In particular, S. mutans biofilms matrix's sticky glucans are known to limit diffusion of CPC and CHX (Bowen and Koo 2011). CPC and CHX biological activity for $S$. mutans biofilms is mainly limited to bactericidal effects rather than to the degradation of glucans or the inhibition of extracellular polymeric substances (EPS) production (Gao et al. 2016; Pandit et al. 2015; Tawakoli et al. 2015). Therefore, the microspray impact might have changed the diffusion-limiting properties of the glucan matrix causing increased delivery. Future work on staining the glucan matrix to assess the effects of microsprays on its structure could be an interesting complement to the bacterial killing and fluorescent beads visualisation.

Another important physiological property of biofilms is the viscoelastic mechanical behaviour (He et al. 2013). Viscoelasticity means the material behaves either as an elastic solid or a viscous fluid depending how fast it is deformed and how quickly it recovers (Banks et al. 2010). The EPS polymers which are kept together by physicochemical interactions (electrostatic interactions, van der Waals forces and hydrogen bonding) appear to be the main contributors to biofilm viscoelasticity (Korstgens 2001). Recent studies on biofilms exposed to a non-contact brushing routine showed a change in viscoelastic properties linked to an increase of antimicrobials penetration (He et al. 2013; He et al. 2014; Jongsma et al. 2015). 
We hypothesize that a combined effect of shear, which left the unremoved biofilm structure in a more vulnerable state enabling better chemical penetration, and antimicrobial delivery yielded an increase in bacteria killing.

Our data showed that the microspray alone resulted in killing of bacteria in the remaining biofilm outside the zone of clearance (Figure 3). It is not clear why this is. Powered and mechanical toothbrushes caused damage to cell surface structures but did not affect the cell viability (McInnes et al. 1993). However, no studies examined viability of biofilm-associated bacteria when exposed to hydrodynamic phenomena. It is possible that the remaining cells could be not completely dead but metabolically compromised and not have the necessary energy reserves to repair essential channels/walls/membranes/receptors. Another possibility is that the microspray could have introduced oxygen into the biofilm causing oxidative stresses. It has been recently demonstrated that presence of oxygen can alters cell surface composition in S. mutans biofilms (Ahn et al. 2007).

In conclusion, low volume high-velocity water microsprays are effective at removing $S$. mutans biofilms from areas relevant to that of a tooth surface and have the additional benefit as a potential delivery method for antimicrobials inside dental biofilm that might remain in or adjacent to the zone of clearance. There are other potential clinical benefits that we did not test but might explain the clinical efficacy of powered interdental devices using high-velocity microsprays in improving gum health (Ward et al. 2015). It is reasonable to assume that if the microspray is effectively mixing the biofilm up to drive fluids into the biofilm then potentially proinflammatory mediators such as bacterial toxins w ould get driven out. Further, dental biofilm is a complex ecosystem made of oxygen-intolerant organisms, it is possible that the turbulent mixing of the biofilm also disrupts the oxygen gradient, and thereby, inhibiting anaerobic growth of periopathogens. It is not still clear what force threshold is required by interdental cleaning devices to produce advective mixing nor what force is required to eliminate biofilms. The further improvement of the synergy between microsprays and antibacterial activity, either by changing the design of the dental device or the use of different drug-delivery methods such as antimicrobial coated nanoparticles or micro/nano emulsions is of interest in future research. 


\section{ACKNOWLEDGMENTS}

This work was financially funded in part by EPSRC DTP EP/K503130/1 award and in part by Philips Oral Healthcare, Bothell, WA, USA. We would like to thank the Southampton Biomedical Imaging Unit for their support in imaging the biofilms. All data supporting this study are openly available from the university of Southampton repository at http://dx.doi.org/10.5258/SOTON/396657.

\section{CONFLICT OF INTEREST}

This work was financially funded in part by EPSRC DTP EP/K503130/1 award and in part by Philips Oral Healthcare, Bothell, WA, USA. M. S. and M. W. are employed by Philips Oral Healthcare, Bothell, WA, USA. M. A was a former employee of Philips Oral Healthcare, Bothell, WA, USA. P. S. has served as a private consultant for POH. The other authors declare no potential conflicts of interest with respect to the authorship and/or publication of this article. 


\section{REFERENCES}

Ahn S-J, Wen ZT, Burne RA. 2007. Effects of oxygen on virulence traits of streptococcus mutans. Journal of bacteriology. 189(23):8519-8527.

Banks HT, Hu S, Kenz ZR. 2010. A brief review of elasticity and viscoelasticity for solids. Adv in Appl Math Mech. 3:1-51.

Bowen WH, Koo H. 2011. Biology of streptococcus mutans-derived glucosyltransferases: Role in extracellular matrix formation of cariogenic biofilms. Caries Research. 45:69-86.

Chew SC, Kundukad B, Seviour T, van der Maarel JRC, Yang L, Rice SA, Doyle P, Kjelleberg S. 2014. Dynamic remodeling of microbial biof ilms by functionally distinct exopolysaccharides. MBio. 5(4):e01536-01514.

Corbin A, Pitts B, Parker A, Stewart PS. 2011. Antimicrobial penetration and efficacy in an in vitro oral biofilm model. Antimicrobial agents and chemotherapy. 55(7):3338-3344.

Costerton JW, Stewart PS, Greenberg E. 1999. Bacterial biofilms: A common cause of persistent infections. Science. 284(5418):1318-1322.

Deshpande MD, Vaishnav RN. 1983. Wall stress distribution due to jet impingement. Journal of Engineering Mechanics. 109(2):479-493.

Dewhirst FE, Chen T, Izard J, Paster BJ, Tanner ACR, Yu W-H, Lakshmanan A, Wade WG. 2010. The human oral microbiome. Journal of bacteriology. 192(19):5002-5017.

Drury WJ, Characklis WG, Stewart PS. 1993a. Interactions of $1 \mu \mathrm{m}$ latex particles with pseudomonas aeruginosa biofilms. Water Research. 27(7):1119-1126.

Drury WJ, Stew art PS, Characklis WG. 1993b. Transport of 1- $\mu \mathrm{m}$ latex particles in pseudomonas aeruginosa biofilms. Biotechnology and Bioengineering. 42(1):111-117.

Fabbri S, Johnston DA, Rmaile A, Gottenbos B, De Jager M, Aspiras M, Starke ME, Ward MT, Stoodley P. 2016. Streptococcus mutans biofilm transient viscoelastic fluid behaviour during high-velocity microsprays. Journal of the mechanical behavior of biomedical materials. 59:197-206.

Gao L, Liu Y, Kim D, Li Y, Hwang G, Naha PC, Cormode DP, Koo H. 2016. Nanocatalysts promote streptococcus mutans biofilm matrix degradation and enhance bacterial killing to suppress dental caries in vivo. Biomaterials. 101:272-284.

Hashizume LN, Dariva A. 2015. Effect of sonic vibration of an ultrasonic toothbrush on the removal of streptococcus mutans biofilm from enamel surface. American journal of dentistry. 28(6):347-350.

He Y, Peterson BW, Jongsma MA, Ren Y, Sharma PK, Busscher HJ, van der Mei HC. 2013. Stress relaxation analysis facilitates a quantitative approach towards antimicrobial penetration into biofilms. PloS one. 8(5):e63750. 
He Y, Peterson BW, Ren Y, van der Mei HC, Busscher HJ. 2014. Antimicrobial penetration in a dualspecies oral biofilm after noncontact brushing: An in vitro study. Clinical Oral Investigations. 18:1103-1109.

Hope C, Wilson M. 2004. Analysis of the effects of chlorhexidine on oral biofilm vitality and structure based on viability profiling and an indicator of membrane integrity. Antimicrobial agents and chemotherapy. 48(5):1461-1468.

Hsu TT, Walker TW, Frank CW, Fuller GG. 2011. Role of fluid elasticity on the dynamics of rinsing flow by an impinging jet. Physics of Fluids (1994-present). 23(3):033101.

Hwang G, Klein MI, Koo H. 2014. Analysis of the mechanical stability and surface detachment of mature streptococcus mutans biofilms by applying a range of external shear forces. Biofouling. 30:1079-1091.

Jongsma MA, van de Lagemaat M, Busscher HJ, Geertsema-Doornbusch GI, Atema-Smit J, van der Mei HC, Ren Y. 2015. Synergy of brushing mode and antibacterial use on in vivo biofilm formation. Journal of dentistry. 43(12):1580-1586.

Koo H, Falsetta ML, Klein MI. 2013. The exopolysaccharide matrix a virulence determinant of cariogenic biofilm. Journal of dental research. 92(12):1065-1073.

Korstgens V. 2001. Influence of calcium ions on the mechanical properties of a model biofilm of mucoid pseudomonas aeruginosa. Water Sci Technol. 43(6):49-57.

Marsh PD. 2004. Dental plaque as a microbial biofilm. Caries Research. 38:204-211.

Marsh PD. 2006. Dental plaque as a biofilm and a microbial community - implications for health and disease. BMC Oral Health. 6 Suppl 1:S14.

McInnes C, Engel D, Martin RW. 1993. Fimbria damage and removal of adherent bacteria after exposure to acoustic energy. Oral microbiology and immunology. 8(5):277-282.

Miller JK, Neubig R, Clemons CB, Kreider KL, Wilber JP, Young GW, Ditto AJ, Yun YH, Milsted A, Badawy HT. 2013. Nanoparticle deposition onto biofilms. Annals of biomedical engineering. 41(1):53-67.

Nance WC, Dowd SE, Samarian D, Chludzinski J, Delli J, Battista J, Rickard AH. 2013. A highthroughput microfluidic dental plaque biofilm system to visualize and quantify the effect of antimic robials. Journal of Antimicrobial Chemotherapy. 68(11):2550-2560.

Pandit S, Cai JN, Jung JE, Lee YS, Jeon JG. 2015. Effect of brief cetylpyridinium chloride treatments during early and mature cariogenic biofilm formation. Oral Diseases. 21(5):565-571.

Phares DJ, Smedley GT, Flagan RC. 2000. The wall shear stress produced by the normal impingement of a jet on a flat surface. Journal of Fluid Mechanics. 418:351-375. 
Rmaile A, Carugo D, Capretto L, Aspiras M, De Jager M, Ward M, Stoodley P. 2014. Removal of interproximal dental biofilms by high-velocity water microdrops. Journal of dental research. 93(1):68-73.

Rmaile A, Carugo D, Capretto L, Wharton JA, Thurner PJ, Aspiras M, Ward M, De Jager M, Stoodley P. 2015. An experimental and computational study of the hydrodynamics of highvelocity water microdrops for interproximal tooth cleaning. Journal of the Mechanical Behavior of Biomedical Materials. 46:148-157.

Stoodley P, A Nguyen D, A Longwell M, A Nistico L, A von Ohle C, A Milanovich N, A de Jager M. 2007. Effect of the sonicare flexcare power toothbrush on fluoride delivery through streptococcus mutans biofilms. Compendium of Continuing Educationin Dentistry. 28:15-22.

Stoodley P, Wefel J, Gieseke A, deBeer D, von Ohle C. 2008. Biofilm plaque and hydrodynamic effects on mass transfer, fluoride delivery and caries. The Journal of the American Dental Association. 139(9):1182-1190.

Tawakoli PN, Ragnarsson KT, Rechenberg DK, Mohn D, Zehnder M. 2015. Effects of endodontic irrigants on biofilm matrix polysaccharides. International endodontic journal.

Vinogradov AM, Winston M, Rupp CJ, Stoodley P. 2004. Rheology of biofilms formed from the dental plaque pathogen streptococcus mutans. Biofilms. 1(01):49-56.

von Ohle C, Gieseke A, Nistico L, Decker EM, deBeer D, Stoodley P. 2010. Real-time microsensor measurement of local metabolic activities in ex vivo dental biofilms exposed to sucrose and treated with chlorhexidine. Applied and Environmental Microbiology. 76:2326-2334.

Walker TW, Hsu TT, Frank CW, Fuller GG. 2012. Role of shear-thinning on the dynamics of rinsing flow by an impinging jet. Physics of Fluids (1994-present). 24(9):093102.

Ward M, Amini P, Fafard T, Olson M, Mwatha A, Jenkins W, Souza S. 2015. Gum health and plaque regrowth response following 4-week interdental hygiene intervention. Paper presented at: IADR/AADR/CADR General Session \& Exhibition. Boston, Massachusetts. 


\section{FIGURES LEGEND}

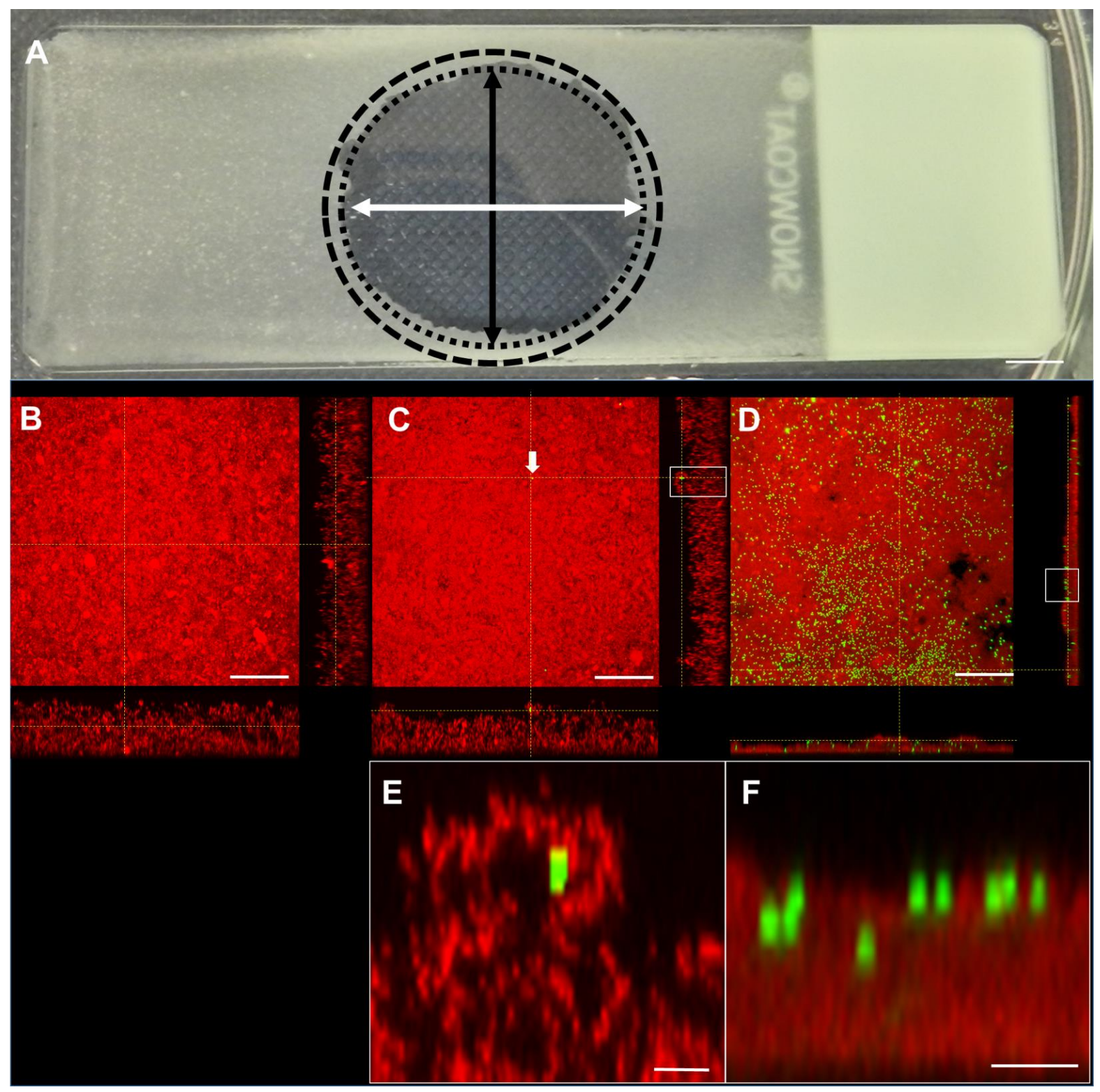

Figure 1. A) Microscope slide covered with $S$. mutans biofilm (light grey) after being exposed to a high velocity PBS microspray at $90^{\circ}$ impact angle. A well-defined circular shaped zone of clearance (ZOC) in the area where biofilm was removed reveals the darker background surface. The black and white arrows show the diameters of the ZOC in the $\mathrm{x}$ - and y-axes respectively (major and minor axes lengths for the $30 \mathrm{o}$ impact). The dotted circle marks the edge of the ZOC and the measurements of transport and killing in the remaining biofilm were made no further than $200 \mu \mathrm{m}$ from outside this edge (dashed circle). Scale bar is $5 \mathrm{~mm}$. Confocal images in $\mathrm{x}-\mathrm{y}$ plan view with $\mathrm{x}-\mathrm{z}$ cross section below of $S$. mutans biofilm without any treatment (control) (B), after the static treatment (C) and after the $90^{\circ}$ impact 
angle microspray (D). $\mathrm{X}-\mathrm{y}$ plan views are maximum projections while the sagittal sections are single slices taken at the transects indicated by the yellow dashed lines on the plan view. Dead biofilm was stained red (Syto 63) and the beads are fluorescent green. Scale bars are 50 $\mu \mathrm{m}$. Close-up views of selected areas (white rectangles) of the confocal cross-sections of the static treatment $(\mathrm{E})$ and the $90^{\circ}$ impact angle microspray $(\mathrm{F})$ showing beads penetration inside the biofilm. Scale bars are $5 \mu \mathrm{m}$.

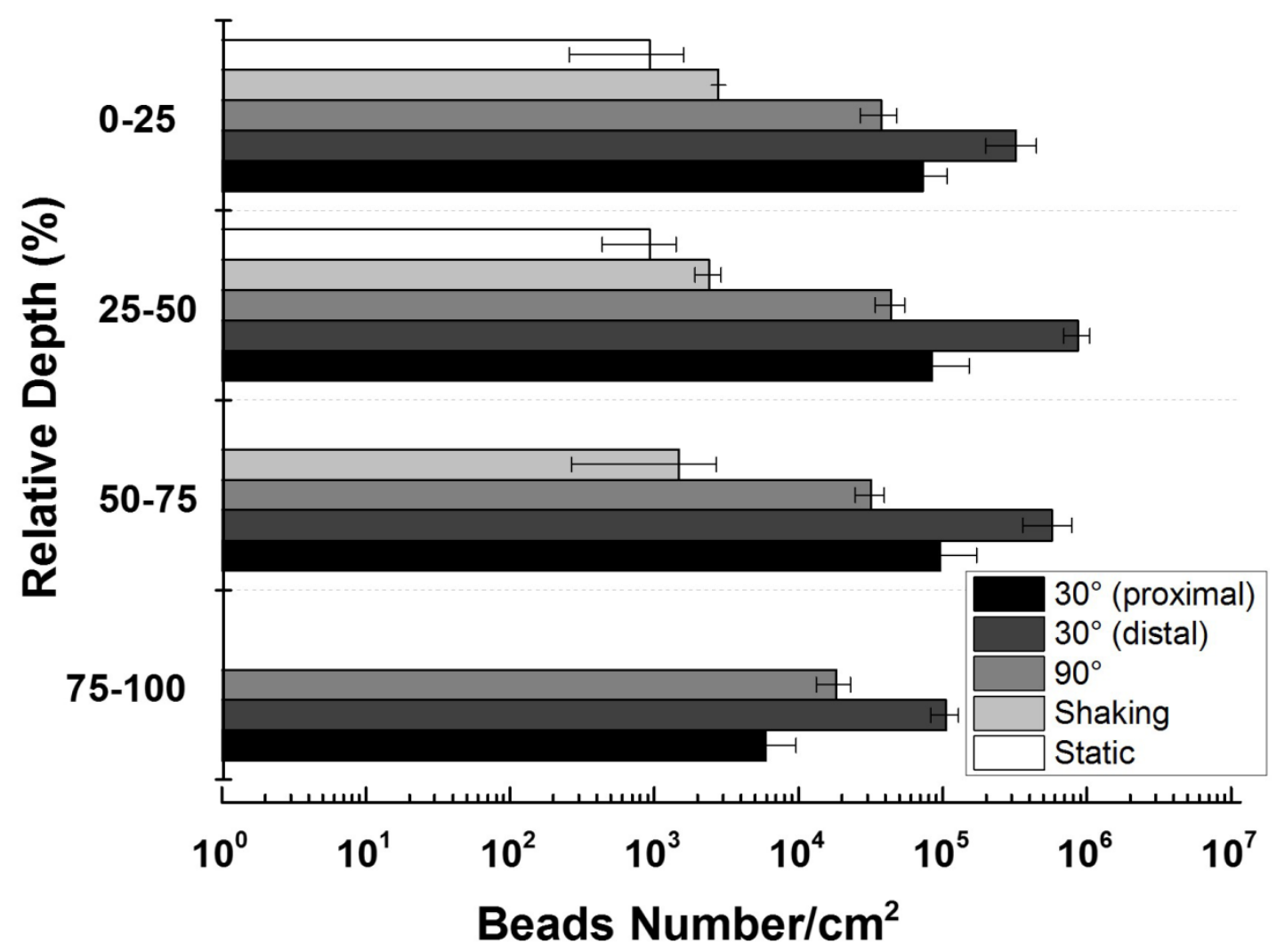

Figure 2. Bar chart (logarithmic scale) showing beads distribution $\left(\mathrm{N} / \mathrm{cm}^{2}\right)$ inside $S$. mutans biofilm in terms of relative depth ratio for the static, shaking, $90^{\circ}$ and $30^{\circ}$ experiments. Data represented as mean and 1SE from three independent replicates. A relative depth value of $0 \%$ corresponded to a bead located near the biofilm surface, while a relative depth of $100 \%$ corresponded to a bead located in the biofilm substratum. 

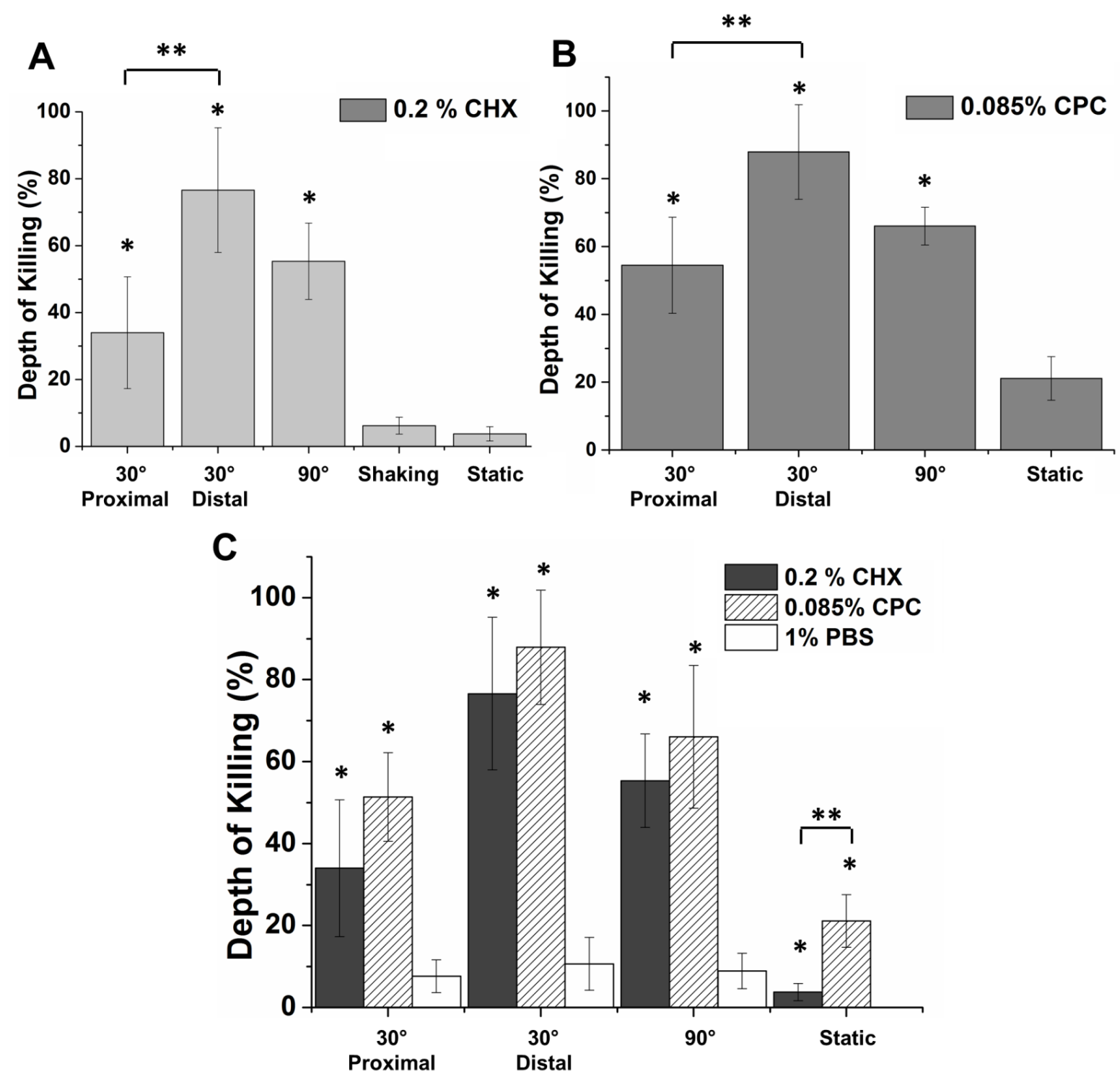

Figure 3. Biofilm depth of killing caused by $0.2 \% \mathrm{CHX}(\mathrm{A})$ or $0.085 \% \mathrm{CPC}(\mathrm{B})$ after the $30^{\circ}$ and the $90^{\circ}$ impact angle microsprays, the static and shaking experiments. Data represented as mean and 1SD from three independent replicates. The single asteris ks represent statistical difference between the microspray experiments $\left(30^{\circ}\right.$ and $90^{\circ}$ impact angles) and the shaking or static assays. Double asterisks represent statistical difference between the $30^{\circ}$ impact angle microspray in the proximal position and the $30^{\circ}$ impact angle microspray in the distal position. C) Biofilm depth of killing caused by $0.2 \% \mathrm{CHX}, 0.085 \%$ and $1 \%$ PBS after the microspray $\left(30^{\circ}\right.$ and $90^{\circ}$ impact angles) and the static treatments. Data represented as mean and 1SD from three independent replicates. The single asterisks represent statistical difference between $0.2 \% \mathrm{CHX}$ or $0.085 \%$ CPC compared to $1 \%$ PBS within the same assay. 
Double asterisks represent statistical difference between the static treatment performed with $0.2 \% \mathrm{CHX}$ and the static treatment performed $0.085 \% \mathrm{CPC}$.

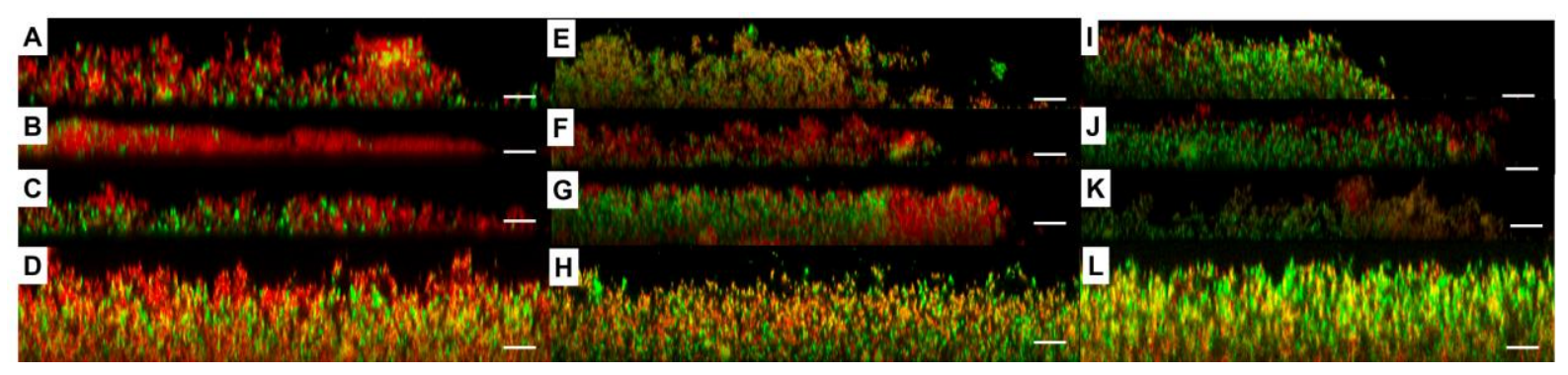

Figure 4. Confocal images in $\mathrm{x}-\mathrm{z}$ cross section view showing $S$. mutans biofilms after the $90^{\circ}$ shooting, $30^{\circ}$ shooting in the distal zone, $30^{\circ}$ shooting in the proximal zone and the static assay using $0.085 \%$ CPC (A-D), 0.2\% CHX (E-F) or 1\% PBS (I-L). Dead biofilm was stained red (Propidium Iodide) and live biofilm green (Syto 9). Scale bars are $20 \mu \mathrm{m}$. 
LIST OF TABLES

\begin{tabular}{|c|c|c|c|c|c|c|c|}
\hline Treatment & \multicolumn{6}{|c|}{ Experiment Exposure } \\
\hline & Static & Shaking & \multicolumn{2}{|c|}{$90^{\circ}$ microspray } & \multicolumn{3}{c|}{$\mathbf{3 0}^{\circ}$ micros pray } \\
\hline & & & $\begin{array}{c}\text { Outside } \\
\text { ZOC }\end{array}$ & ZOC & \multicolumn{2}{|c|}{ Outside ZOC } & ZOC \\
\hline & & & & & Proximal & Dis tal & \\
\hline 1\% PBS & $45.9 \pm 6.3$ & - & $28.1 \pm 4.9$ & $0.005 \pm 0.003$ & $20.1 \pm 4.7$ & $26.1 \pm 3.9$ & $0.006 \pm 0.001$ \\
\hline Microbeads & $55.6 \pm 1.1$ & $43.9 \pm 2.6$ & $24.7 \pm 2.8$ & $0.005 \pm 0.002$ & $25.6 \pm 7.9$ & $21.4 \pm 4.1$ & $0.007 \pm 0.003$ \\
\hline $\mathbf{0 . 2 \%}$ CHX & $51.4 \pm 3.6$ & $43.6 \pm 1.9$ & $28.3 \pm 4.6$ & $0.007 \pm 0.004$ & $20.5 \pm 3.5$ & $18.3 \pm 1.5$ & $0.004 \pm 0.002$ \\
\hline $\mathbf{0 . 0 8 5 \%}$ CPC & $39.8 \pm 8.4$ & - & $21.9 \pm 7.3$ & $0.004 \pm 0.002$ & $18.3 \pm 1.4$ & $18.9 \pm 3.4$ & $0.008 \pm 0.003$ \\
\hline
\end{tabular}

Table 1. Average biofilm thickness $(\mu \mathrm{m})$ as a function of the various exposures and treatment solutions. Data represented as mean and 1 SD from three independent replicates. 


\section{APPENDIX FIGURES}
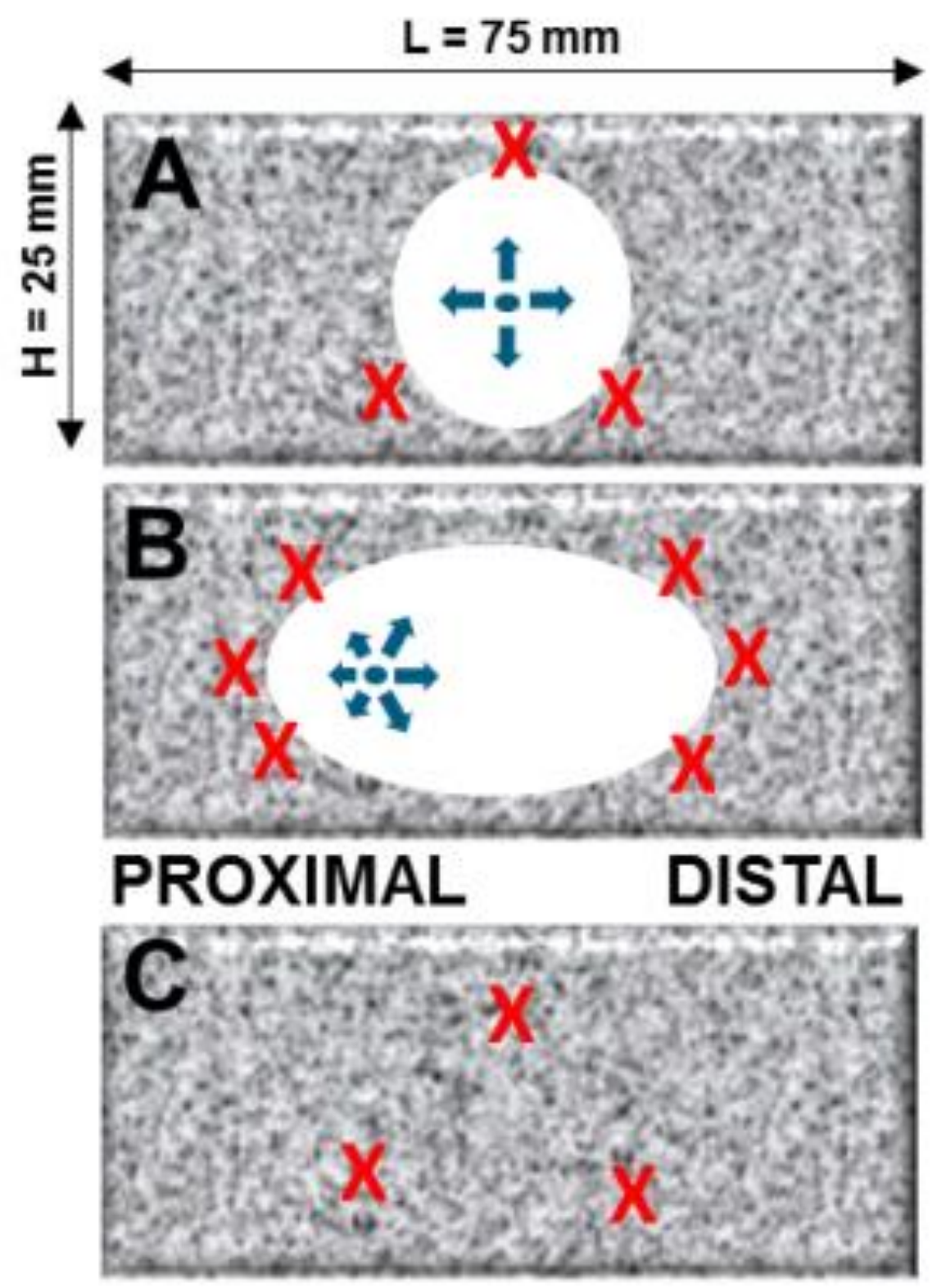

Appendix Figure 1. Representation of one biofilm-covered slide (biofilm depicted as grey and the zone of clearance (ZOC) as white) showing the confocal images positions taken for the $90^{\circ}$ impact angle microspray (A), the $30^{\circ}$ impact angle microspray (B) and the control, diffusion and shaking experiments $(\mathrm{C})$. The proximal zone refers to the ZOC behind the point of impact (with respect to the direction of the flow) whereas the distal zone to the ZOC on the opposite side of the proximal zone. Images were taken 1-2 mm after the edge of the zone of clearance. Blue arrows show the direction of the flow from the impact centre. 

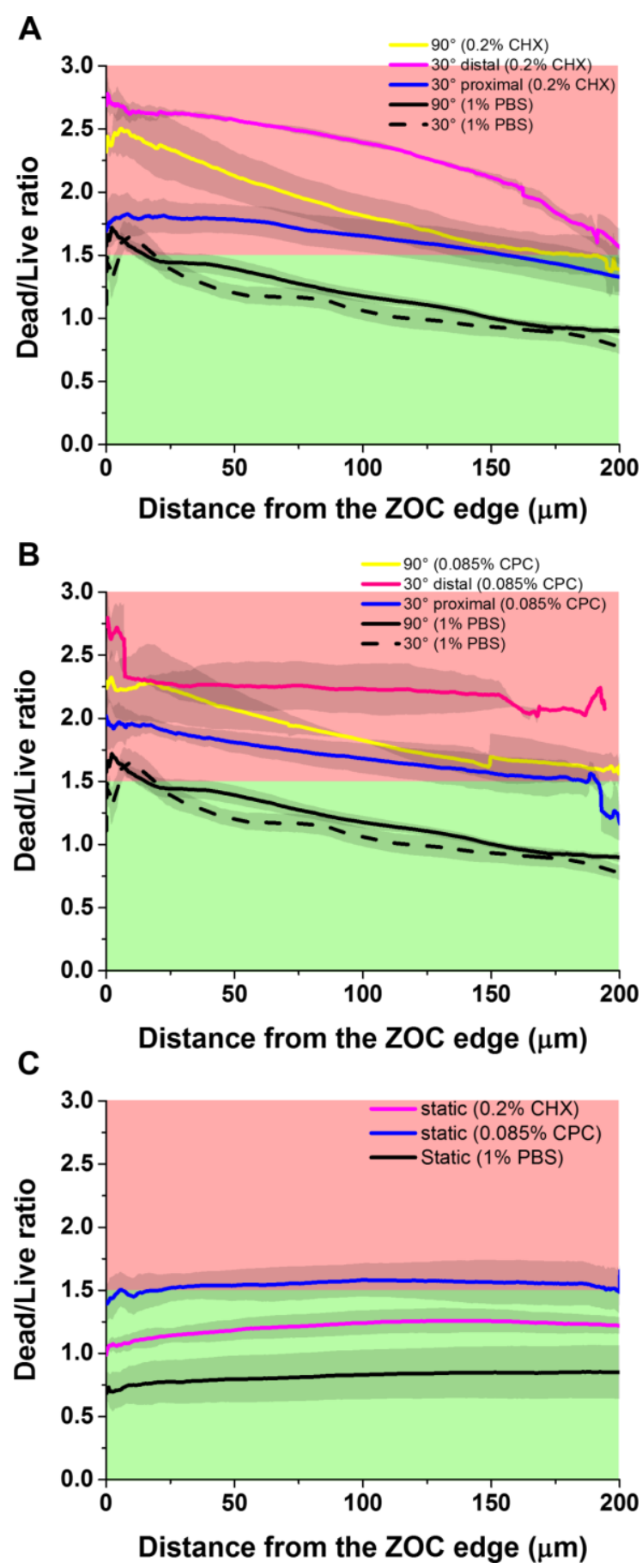

Appendix Figure 2. Biofilm dead/live ratio as a function of the lateral distance moving outwards from the edge of the ZOC (see Figure 1) caused by the microspray exposure ( $30^{\circ}$ and $90^{\circ}$ impact angles) when using $0.2 \% \mathrm{CHX}(\mathrm{A})$ or $0.085 \% \mathrm{CPC}$ (B) and caused by the static diffusion exposure to $0.2 \%$ CHX, $0.085 \%$ CPC and 1\% PBS (C). The solid lines are the average dead/live ratio and the coloured areas are SD intervals from three independent replicates. Below the threshold ratio of 1.5 biofilm was considered "alive" (green background) while above 1.5, biofilm was considered "dead" (red background). 


\section{SUPPLEMENTAL MATERIAL}

The microsprays generated a zone of clearance (ZOC) in the area where the biofilm was removed (Figure 1A). The $90^{\circ}$ impact angle generated a circular ZOC of approximately 300 $\mathrm{mm}^{2}$, as the flow pattern was symmetric. In contrast, the $30^{\circ}$ impact angle created an elliptical ZOC of approximately $450 \mathrm{~mm}^{2}$ because the liquid flow over the surface became asymmetric and less biofilm was removed behind the point of impact (proximal zone, with respect to the direction of the flow). For the spray-exposed biofilm slides $\left(90^{\circ}\right.$ and $30^{\circ}$ impact angles) confocal images were taken 1-2 mm outside the zone of clearance to represent the unimpacted biofilm. For the $90^{\circ}$ impact angle spray, images where taken around the circular zone (Appendix Figure 1A). As for the $30^{\circ}$ impact angle spray, and to assess whether the symmetry of the flow influenced beads and/or antimicrobial penetration, confocal images were taken at the proximal and at the distal positions (Appendix Figure 1B). Confocal images were also taken inside the zone of clearance (interior zone). For the static and shaking experiments, confocal images where taken on the exposed area of the sample (Appendix Figure 1C). To establish the thickness of the biofilm prior the treatments, confocal images were also taken on the control biofilm slides (Appendix Figure 1C). The thickness of the control biofilms was measured by COMSTAT program (http://www.comstat.dk/, (Heydorn et al. 2000)) using confocal images.

\section{Supplemental material 2}

The relative depth ratio $\left(\mathrm{RD}_{\mathrm{BEADS}}\right)$ was defined as

$$
R D_{B E A D S}(\%)=\left(1-\frac{\left.z_{B E A D} x_{i}, y_{i}\right)}{z_{B I O F I L M}\left(x_{i}, y_{i}\right)}\right) \times 100
$$

where $\mathrm{z}_{\mathrm{BEAD}}$ was the bead z-coordinate (depth) and $\mathrm{z}_{\mathrm{BIOFILM}}$ was the biofilm thickness relative to each bead position $\left(\mathrm{x}_{\mathrm{i}}, \mathrm{y}_{\mathrm{i}}\right)$. A RD $=0 \%$ corresponded to a bead located near the biofilm surface, while a RD $=100 \%$ corresponded to a bead located in the biofilm substratum adjacent to the glass slide. $\mathrm{Z}_{\mathrm{BEAD}}$ values were measured using Fiji (http://fiji.sc/Fiji) (Schindelin et al. 2012) while $\mathrm{Z}_{\mathrm{BIOFILM}}$ ones were obtained using COMSTAT. The beads number $(\mathrm{N})$ in each confocal image was measured using the Analyse Particles function of Fiji. The areal density of beads were measured per $\mathrm{cm}$ square area and normalized by the area of one confocal image which was approximately $0.6 \times 10^{-3} \mathrm{~cm}^{2}$. 


\section{Supplemental material 3}

Confocal images were analysed with Fiji. Using the Plot Profile function on an $\mathrm{x}-\mathrm{z}$ cross section view, the ratio of the intensity of red to green $(R / G)$ as a function of the biofilm thickness was obtained. Five random measurements were taken on each cross-section for the diffusion, shaking and microspray experiments. A biofilm dead band was defined when R/G became less than 1.5 (He et al. 2014). Next, CHX and CPC killing depth ratios $\left(\mathrm{KD}_{\mathrm{CHX}}\right.$ and $\mathrm{KD}_{\mathrm{CPC}}$ ) were calculated as:

$$
\mathrm{KD}=\frac{\text { dead band thickness }}{\text { biofilm thickness }} \times 100 \%
$$

\section{Supplemental material 4}

The $x-y$ plan views of the confocal images were analysed with Fiji measuring the average projection of the live and dead channels to obtain an averaged (along $\mathrm{z}$ ) dead or live intensity for each point of the $\mathrm{x}-\mathrm{y}$ view. For those biofilms exposed to the microsprays, we assessed the changes in the red or green, respectively, intensity along the lateral distance from the edge of the zone of clearance by using the Plot profile function. Then the ratio between red/ green was calculated and plotted vs the distance (Appendix Figure 2).

\section{REFERENCES}

He Y, Peterson BW, Ren Y, van der Mei HC, Busscher HJ. 2014. Antimicrobial penetration in a dualspecies oral biofilm after noncontact brushing: An in vitro study. Clinical Oral Investigations. 18:1103-1109.

Heydorn A, Nielsen AT, Hentzer M, Sternberg C, Givskov M, Ersbøll BK, Molin S. 2000. Quantification of biofilm structures by the novel computer program comstat. Microbiology. 146(10):2395-2407.

Schindelin J, Arganda-Carreras I, Frise E, Kaynig V, Longair M, Pietzsch T, Preibisch S, Rueden C, Saalfeld S, Schmid B et al. 2012. Fiji: An open-source platform for biological-image analysis. Nat Methods. 9(7):676-682. 